\title{
Interfacial Charge Transfer in Nanoscale Polymer Transistors
}

\author{
Jeffrey H. Worne ${ }^{1}$, Rajiv Giridharagopal ${ }^{1}$, Kevin F. Kelly ${ }^{1}$, and Douglas Natelson ${ }^{1,2}(\square)$ \\ ${ }^{1}$ Department of Electrical and Computer Engineering, Rice University, 6100 Main St., Houston, TX 77005, USA \\ ${ }^{2}$ Department of Physics and Astronomy, Rice University, 6100 Main St., Houston, TX 77005, USA \\ Received: 31 July 2008/ Revised: 1 September 2008/Accepted: 1 September 2008 \\ OTsinghua Press and Springer-Verlag 2008. This article is published with open access at Springerlink.com
}

\begin{abstract}
Interfacial charge transfer plays an essential role in establishing the relative alignment of the metal Fermi level and the energy bands of organic semiconductors. While the details remain elusive in many systems, this charge transfer has been inferred in a number of photoemission experiments. We present electronic transport measurements in very short channel $(L<100 \mathrm{~nm})$ transistors made from poly(3-hexylthiophene) (P3HT). As channel length is reduced, the evolution of the contact resistance and the zero gate voltage conductance are consistent with such charge transfer. Short channel conduction in devices with Pt contacts is greatly enhanced compared to analogous devices with Au contacts, consistent with charge transfer expectations. Alternating current scanning tunneling microscopy (ACSTM) provides further evidence that holes are transferred from Pt into P3HT, while much less charge transfer takes place at the Au/ P3HT interface.
\end{abstract}

\section{KEYWORDS}

Organic semiconductors, band alignment, charge transfer, organic field-effect transistor, scanning tunneling microscopy

Understanding the band alignment between organic semiconductors (OSCs) and metal electrodes is of basic physical interest as well as significant technological importance [1]. Such energetic considerations are crucial for optimizing charge injection in organic light emitting diodes (OLEDs) and organic field-effect transistors (OFETs). Similarly, the relative band alignment at such interfaces also affects the open circuit photovoltage achievable in organic photovoltaic applications. Because interfacial dipoles can be used to modulate the effective work function of a metal surface, self-assembled monolayers (SAMs) of polar molecules have been used in both OLEDs and OFETs to engineer charge injection [2-4].

Conceptually, the issue is straightforward, though very complicated in detail. In equilibrium the chemical potential throughout a metal/organic heterointerface must be constant. This condition is achieved through a combination of interfacial dipole formation, charge transfer, and the self-consistent solution of the electrostatics to equilibrate carrier drift and diffusion. Dipole formation and charge transfer lead to deviations from the Schottky-Mott limit, so that simple alignment of vacuum levels does not give an accurate picture of the true heterojunction energetics [5].

The relative alignment of levels is most readily

Address correspondence to natelson@rice.edu 
measured via photoemission experiments. Recent investigations using electroluminescent polymers [6] as well as small molecule OSCs and conducting polymers [7] have shown Fermi level pinning at the metal-organic interface. For the case of holes, when the Fermi level of the metal is relatively far above the highest occupied molecular orbital (HOMO) of the molecules (the center of the disorder-broadened valence band, in the case of polymers), the system is in the Schottky-Mott limit. When the Fermi level approaches the $\mathrm{HOMO}$, strong deviations from the Schottky-Mott limit have been reported [6]. There are several suggested mechanisms for these deviations, including changes in the interfacial dipole (via chemical reactions or the "pillow effect" [8]), shifting of frontier orbitals and formation of induced states [9], and charge transfer involving polaron formation $[6,10]$. Recent photoemission experiments examining metal/polymer interfaces show evidence of charge transfer into valence band tail states and accompanying band bending [11].

Other data consistent with charge transfer and band bending at similar interfaces have been seen in transport measurements $[4,16]$ of contact resistance in P3HT-based OFETs. In devices with high electrode work functions, hole injection remains ohmic down to very low carrier densities, a natural result if interfacial charge transfer effectively dopes the contact interface. Similarly current-voltage characteristics in devices with (lower work function) electrode metals giving non-ohmic injection are consistent with a nanoscale region (between 10 $\mathrm{nm}$ and $100 \mathrm{~nm}$ in extent) of reduced mobility at the metal/organic interface [12-15]. This may be due to interfacial structural disorder in the organic semiconductor, but it would also be compatible with the formation of an effective depletion region near the contacts due to band bending. Conduction in P3HT takes place via hopping through a density of localized states in the valence band tail. A local change in the chemical potential farther into the band tail would simultaneously deplete holes and reduce the spatial density of available hopping sites, reducing the mobility as well.

In this paper we present additional evidence that significant charge transfer takes place at the
Pt/P3HT interface, leading to a population of mobile holes within a nanoscale distance of the interface. Measurements of Pt-based OFET device resistance as a function of channel length, $L$, show a decrease in resistance as $L$ is reduced below $100 \mathrm{~nm}$. In the same devices this corresponds to a factor of nearly 10 increase in zero gate bias (ZGB) current over the same $L$ range. In contrast, identically prepared short channel Au-based devices show an increase in resistance, as well as ZGB currents orders of magnitude lower than in Pt devices. Devices with $\mathrm{Au}$ surfaces functionalized with SAMs known to increase the electrode effective work function show trends intermediate between the $\mathrm{Au}$ and Pt cases. Further measurements using alternating current scanning tunneling microscopy (ACSTM) show clear evidence for excess holes in thin P3HT films on Pt, in marked contrast to ACSTM measurements on identically prepared P3HT films on Au.

Devices were fabricated on n-silicon with $200 \mathrm{~nm}$ of thermally grown oxide as the gate dielectric. Electrodes were patterned using electron beam lithography, e-beam evaporation, and liftoff processing. Using two-step lithography, various channel lengths, $L$, were fabricated, from $\sim 50 \mathrm{~nm}$ to $5 \mu \mathrm{m}$, with widths, $w$ of $50 \mu \mathrm{m}$ for short channel device studies. In addition, for comparison a structure of interdigitated electrodes with $L=1-50 \mu \mathrm{m}$ and $w=200 \mu \mathrm{m}$ was fabricated on the same chip in order to allow standard transmission line measurements of P3HT field effect mobility. Electrode films were $15 \mathrm{~nm}$ of either gold or platinum with a 1-nm titanium adhesion layer. Following lithography and liftoff, the devices were cleaned under ultraviolet exposure, 1 min of oxygen plasma, and a 1 min soak in piranha etch $\left(3: 1 \mathrm{H}_{2} \mathrm{SO}_{4}\right.$ : $\mathrm{H}_{2} \mathrm{O}_{2}(30 \%)$ ). For functionalized Au electrode devices, the piranha etch step was followed by self-assembly of fluorinated oligo(phenylene ethynylene) (F-OPE) [4] by immersion for $24 \mathrm{~h}$ in a solution of F-OPE at $0.25 \mathrm{mg} / \mathrm{mL}$ concentration in 1:1 ethanol:chloroform under nitrogen gas, with standard thioacetate deprotection chemistry [17].

After the $\mathrm{SiO}_{2}$ dielectric surface was treated with octadecyltrichlorosilane (OTS) $(10 \mu \mathrm{L}$ OTS in $8 \mathrm{~mL}$ hexadecane, assembled in the dark at room temperature for $\sim 12 \mathrm{~h})$, P3HT $(0.1 \%$ by mass in 
chloroform) was then spin-coated to a thickness of 5 $\mathrm{nm}$ (measured via atomic force microscopy (AFM)) over the entire substrate. Contact to the substrate is made, and the Si becomes the gate of the device. Figure 1(b) illustrates a typical short channel device. Devices were transferred to a variable temperature vacuum probe station immediately upon completion of spin coating and remained in vacuum $\left(\sim 10^{-6} \mathrm{mTorr}\right)$ for at least $1 \mathrm{~h}$ before any measurements were performed. Individual OFETs were isolated from one another by using a probe tip to scratch away the P3HT film in a rectangle around each device to prevent stray current paths.

Using the interdigitated electrodes of fixed $w$ but varying $L$, device characteristics $\left(I_{\mathrm{D}} \mathrm{vs} V_{\mathrm{SD}}\right.$ at fixed gate voltage, $V_{\mathrm{G}}$, where $I_{\mathrm{D}}$ is drain current and $V_{\mathrm{SD}}$ is source-drain voltage) were measured via a semiconductor parameter analyzer. In the shallow channel regime $\left(\left|V_{\mathrm{SD}}\right|<<\left|V_{\mathrm{G}}\right|\right)$ we find these characteristics to be linear, consistent with Ohmic injection. As in our previous work $[4,18]$ we use the transmission line approach to characterize the contact resistances and field-effect mobilities as a function of $V_{\mathrm{G}}$. In the shallow channel regime at a particular $V_{\mathrm{G}}$ the measured source-drain resistance $R=\left(\partial I_{\mathrm{D}} / \partial V_{\mathrm{SD}}\right)^{-1}$ was plotted as a function of $L$ and found to be linear for long $(L>1 \mu \mathrm{m})$ devices. Channel resistance, $R_{\mathrm{C}}$, is determined by the slope of this line and the effective contact resistance, $R_{\mathrm{S}}$ is determined by extrapolating this line to zero channel length, as shown in Fig. 1(a). This linearity observed in longer devices indicates that the overall film uniformity is good, even for the relatively thin P3HT layers used here.

This extrapolation to infer $R_{\mathrm{S}}$ assumes that the device properties are uniform down to arbitrarily short channel lengths. In practice this may not be true, for a number of reasons. For example, a change in polymer morphology near the metal contacts would lead to deviations from linearity in $R(L)$ at short distances as contact regions interact with each other [19]. Similarly, there is indirect evidence [1214] that nanoscale regions of poor effective mobility can exist near contacts due to band bending and depletion effects. Note that the characteristic size of these regions is constrained experimentally [14] to less than $\sim 100 \mathrm{~nm}$, since larger regions would

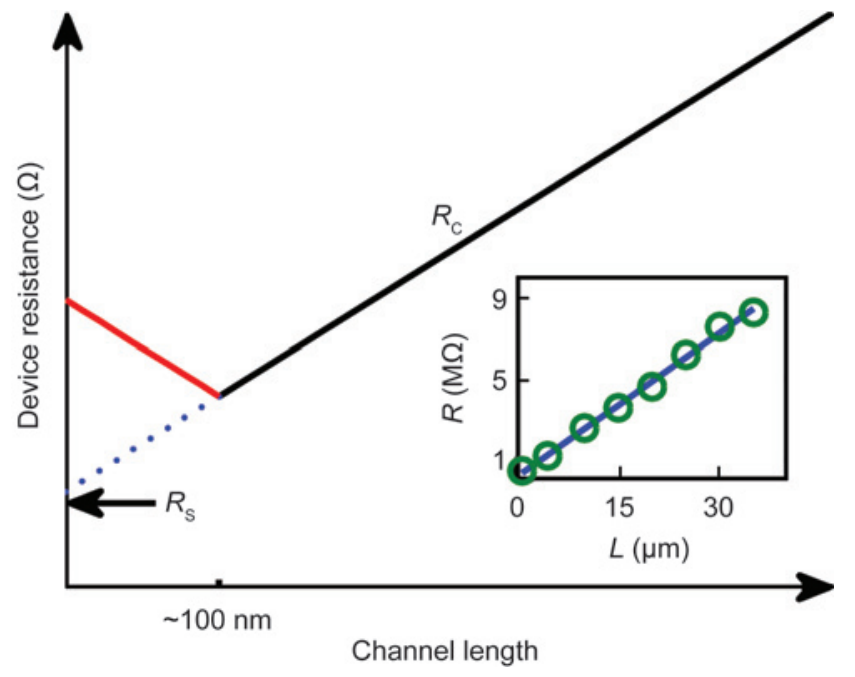

(a)

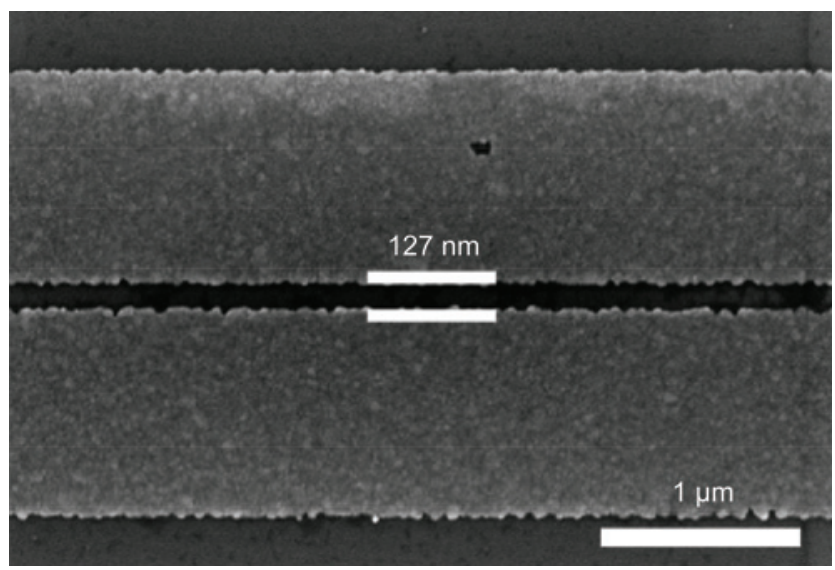

(b)

Figure 1 (a) Expected two-terminal resistance as a function of channel length, with deviations at very short channels expected if contact interface regions have properties that differ significantly from the bulk. Inset: data on a series of devices with channel width 200 $\mu \mathrm{m}$ at $V_{\mathrm{G}}=-50 \mathrm{~V}$ and $T=300 \mathrm{~K}, V_{\mathrm{SD}}$ from 0 to $-1 \mathrm{~V}$, showing the long-channel dependence. Of interest in our experiments is the very short channel limit. (b) Electron micrograph of the center of a typical short channel device fabricated by electron beam lithography

be detectable within the resolution of scanning potentiometry experiments. If two such depletion regions were to intersect for sufficiently short channel devices, one would expect the measured $R(L)$ to have an up-turn as $L \rightarrow 0$, as shown by the red line in Fig. 1(a).

Figure 2 shows these $R$ vs $L$ plots for three sets of $\mathrm{Au}, \mathrm{F}-\mathrm{OPE} / \mathrm{Au}$, and Pt devices at $300 \mathrm{~K}$ and $V_{\mathrm{G}}=-70 \mathrm{~V}$ for shorter channel devices. The resistance was measured via $V_{\mathrm{SD}}$ sweeps from 0 to $-500 \mathrm{mV}$, while gate voltages from $0 \mathrm{~V}$ to $-70 \mathrm{~V}$ (in $10 \mathrm{~V}$ steps) were examined. The mobilities at $V_{\mathrm{G}}=-70 \mathrm{~V}$, inferred from 


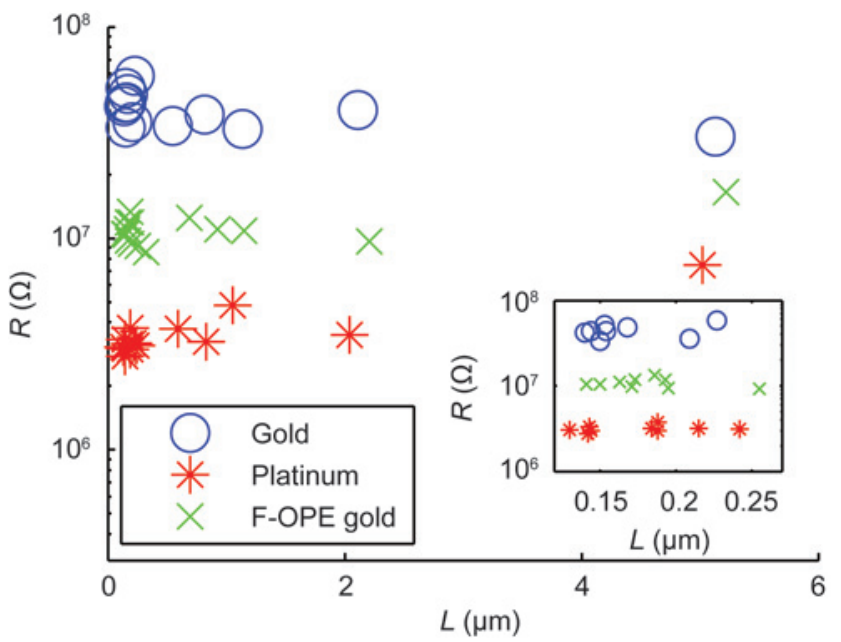

Figure 2 Two-terminal resistance at low $V_{S D}$ at $300 \mathrm{~K}$ and $V_{G}=$ $-70 \mathrm{~V}$ for various series of OFETs incorporating different electrode materials. Note the greatly enhanced resistance in very short channel Au devices compared with Pt devices. Inset shows a more clear view of the short channel data

the length dependences, were $5.3 \times 10^{-2} \mathrm{~cm}^{2} /(\mathrm{V} \cdot \mathrm{s})$ $(\mathrm{Au}), 5.3 \times 10^{-2} \mathrm{~cm}^{2} /(\mathrm{V} \cdot \mathrm{s})(\mathrm{F}-\mathrm{OPE} / \mathrm{Au})$, and $8.7 \times$ $10^{-2} \mathrm{~cm}^{2} /(\mathrm{V} \cdot \mathrm{s})(\mathrm{Pt})$. Initial measured resistances from the $5 \mu \mathrm{m}$ channel length devices span roughly an order of magnitude between the three different electrode materials, with the highest resistance coming from the gold electrodes and the lowest resistance from the platinum devices. As the channel length is reduced significantly below $1 \mu \mathrm{m}$, there are qualitative differences between the three types of devices. The Au-based devices have much increased resistances, while the Pt-based devices have decreased resistances. The F-OPE/ $\mathrm{Au}$ structures are intermediate in their small $L$ properties. We analyzed several ensembles of identically prepared devices, and all exhibited these trends. These trends continued to hold at other gate voltages and as $T$ was reduced. Channel lengths in the short channel devices were measured via electron microscopy after electrical characterization, to ensure that electrode contamination during imaging did not affect the results.
At the smallest channel lengths there is significant device-to-device variability in $R$, much more so than in the longer channel devices. This appears to indicate that microscopic differences in the metal/ organic interface can have significant local impact on the injection process and contact resistances, even when large-scale properties are uniform and well defined. However, even with this variability the overall trends and the systematic deviation between the different electrode types are clear.

One possibility is that the morphology of the P3HT may differ at the metal/ P3HT interface for the three different electrode materials, leading to varying contact properties. To test this, we performed tapping-mode AFM scans of a $3 \mu \mathrm{m} \times 3 \mu \mathrm{m}$ area that encompassed the electrode/P3HT interface for both platinum and gold electrodes. The results are shown in Fig. 3. The topographic images show that the polymer film on the oxide adjacent to the electrode edge is smooth ( $\mathrm{rms}$ roughness of $0.83 \mathrm{~nm}$ for the P3HT on oxide next to $\mathrm{Au}$; $0.78 \mathrm{~nm}$ for the P3HT on oxide next to $\mathrm{Pt}$ ) and largely featureless for both electrode materials. Using phase imaging,

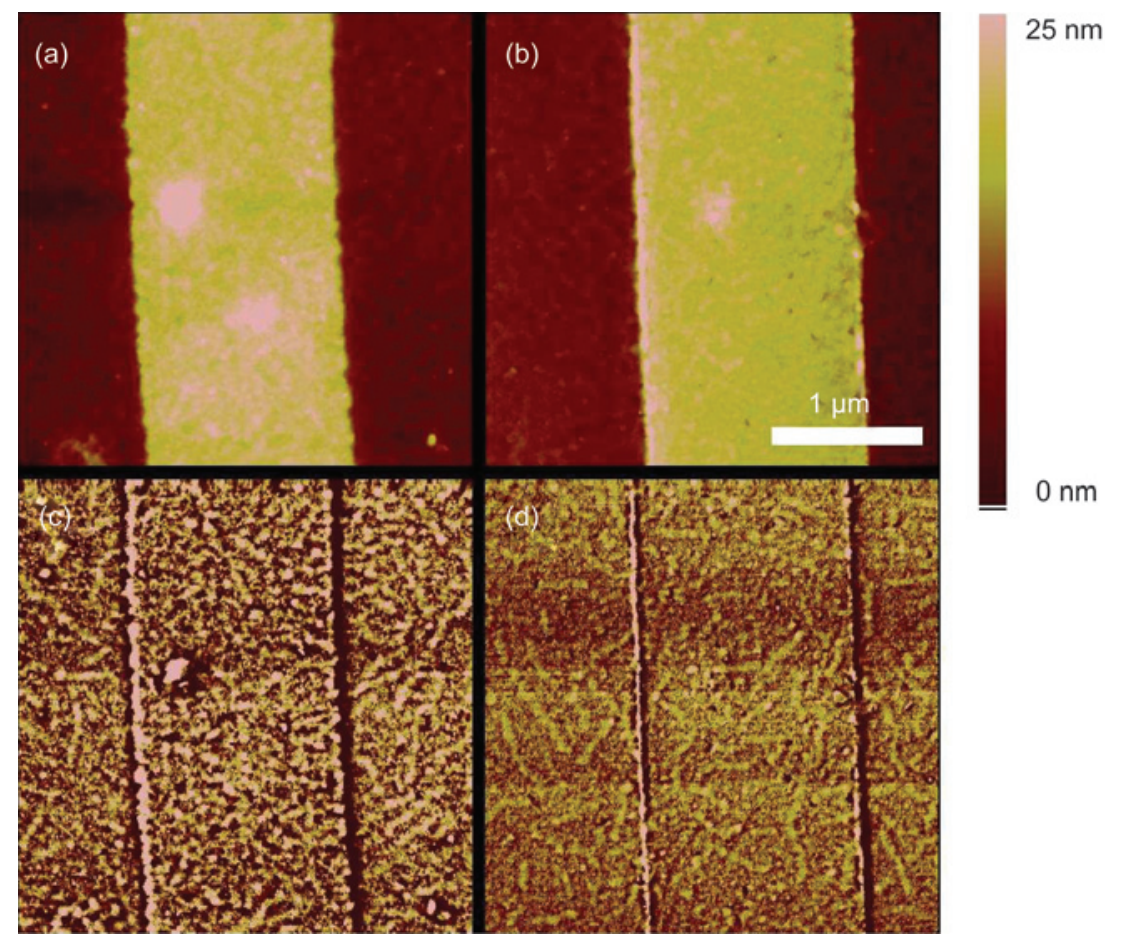

Figure 3 Atomic force microscopy images of P3HT films in contact with gold (a), (c) and platinum (b), (d) electrodes. There is no significant difference in P3HT morphology at the two interfaces, suggesting that the observed differences in electrical properties do not result from greatly varying amounts of disorder 
more detail is observable, with some indications of the fibril morphology sometimes observed [20] in P3HT. However, there is little difference between the two images, at least down to the resolution of our microscope. This implies that gross morphology changes near the contacts are not responsible for the difference in contact resistance properties.

We suggest instead that the explanation for the difference lies in interfacial charge transfer between the electrodes and the P3HT due to the energetics of band alignment. Previous experiments [4, 16, 21] have suggested that in the absence of unintentional doping from the environment the alignment between the Au Fermi level and the P3HT HOMO is such that a significant hole injection barrier exists. In this case one may expect the tail of the P3HT valence band to be locally depleted in the vicinity of the interface. In contrast, measurements involving the interface between P3HT and higher work function systems such as Pt [4, 16] and F-OPE/Au [4] show much lower contact resistances and persistent ohmic injection in field-effect structures even under treatment conditions where the bulk two-terminal conductivity of the P3HT is immeasurably small. Consistent with recent photoemission experiments [11], we suggest that the charge transfer responsible for pinning the Pt Fermi level above the P3HT HOMO populates the tail states with mobile holes, leading to comparatively enhanced conduction in the shortest devices.

This interpretation is supported by other experimental signatures. First, we examine the low $V_{\mathrm{SD}}$ transistor characteristics as a function of $V_{\mathrm{G}}$ for short channel devices with identical geometries. This is more revealing than $I_{\mathrm{D}}-V_{\mathrm{G}}$ transfer characteristics since the changing channel geometry as $L$ is reduced, high electric fields at large $V_{\mathrm{SD}}$ and hysteresis in $V_{\mathrm{G}}$ complicate the interpretation of inferred threshold voltages. A typical result is shown in Fig. 4, for $\mathrm{Pt}$ (a) and $\mathrm{Au}(\mathrm{b})$ devices with $L \approx 100 \mathrm{~nm}$ at $T=300 \mathrm{~K}$. Note that there is a large qualitative difference between these devices. The Pt device shows significant background conduction at $V_{\mathrm{G}}=0$, qualitatively similar to what is seen in the presence of doping. This conduction vanishes in adjacent, simultaneously fabricated Pt devices as channel lengths are increased

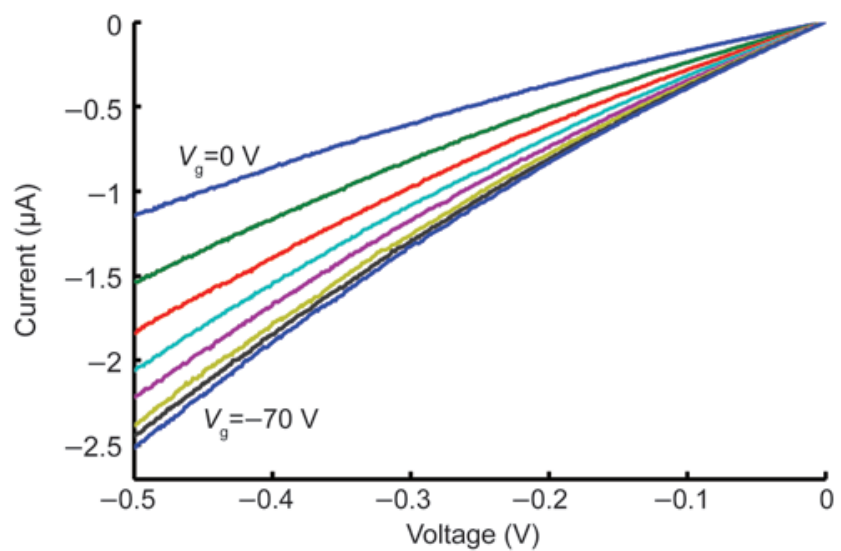

(a)

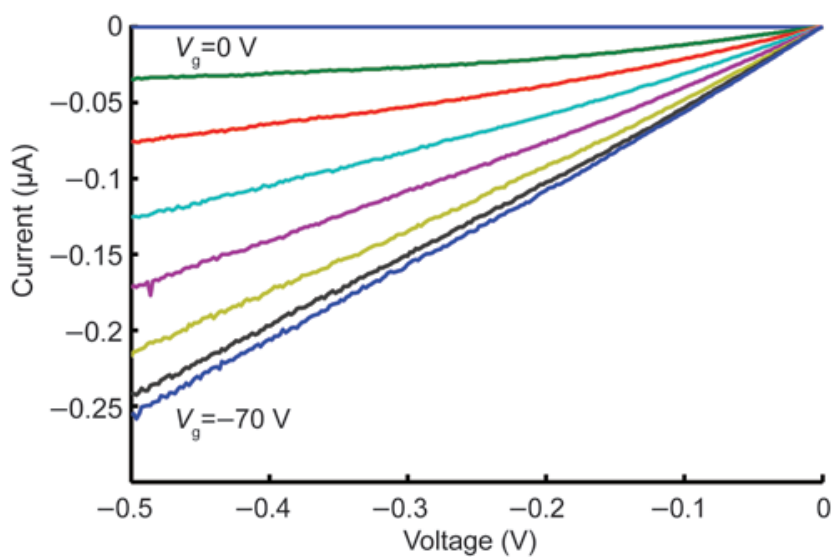

(b)

Figure 4 Transistor characteristics at low $V_{S D}$ for identically prepared Pt (a) and Au (b) devices with channel lengths $\sim 144 \mathrm{~nm}$ and width $50 \mu \mathrm{m}$ at $T=300 \mathrm{~K}$. These devices have essentially identical geometries, yet the Pt-based device shows significant background conduction even at $V_{G}=0$. This qualitative difference vanishes as channel lengths exceed the few micron scale

into the micron range, showing that it is an effect of the contacts.

We investigate this further by examining the twoterminal source-drain conductance at $V_{\mathrm{G}}=0$ for the short channel devices $(L<5 \mu \mathrm{m})$ at $V_{\mathrm{SD}}=-0.5 \mathrm{~V}$. Representative data are shown in Fig. 5. At the $5 \mu \mathrm{m}$ limit the ZGB current is essentially the same for all devices. As the channel length decreases, however, the measured currents in devices with platinum contacts continue to increase by orders of magnitude, while the measured currents in devices with gold contacts do not follow this trend. Rather, for the $\mathrm{Au}$ device $V_{\mathrm{G}}=0$ currents tend to be fairly constant over the measured channel length range. The current measured from the F-OPE/Au electrodes remains fairly constant and similar to that of gold over the

\section{量 Springer}




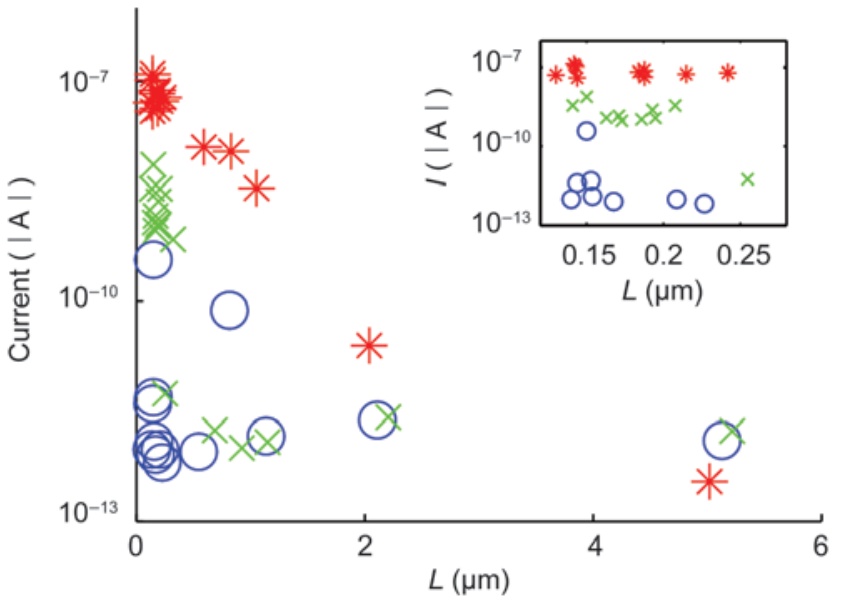

(a)

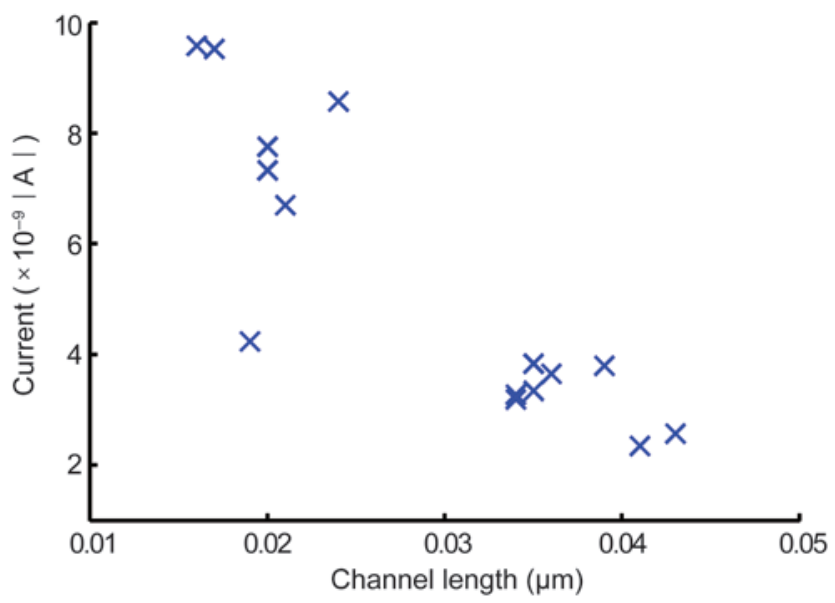

(b)

Figure 5 (a) ZGB current (measured at $V_{G}=0, V_{S D}=-0.5 \mathrm{~V}$ ) at $300 \mathrm{~K}$ for the series of devices (same symbols) in Fig. 2. Notice the great enhancement in ZGB current for extremely short channel Pt and F-OPE/Au devices, much larger than simple geometric expectations would suggest. (b) Data acquired in a separately fabricated ensemble of Pt-based devices made using the nanogap method of Ref. [29]

longer channel lengths, but as the channel lengths decrease well below $200 \mathrm{~nm}$, the zero bias current displays a rapid increase. Since all of the devices of a given electrode composition are prepared simultaneously and measured in one run, these dependences of the $V_{\mathrm{G}}=0$ conduction on $L$ cannot arise from dopants from the environment.

To further examine charge transfer between the electrodes and the P3HT film, we used an extension of scanning tunneling microscopy (STM) known as alternating current Scanning electron microscopy (ACSTM). STM and related spectroscopic techniques have long been used to characterize the electronic properties of conducting polymer materials. More common spectroscopies provide high resolution analysis of properties such as the density of states and work function, yet fail to provide capacitance versus voltage $(C-V)$ information, which is critical for understanding dopant effects. Unlike conventional STM, microwave frequency ACSTM can be used to obtain local $C-V$ data.

In the ACSTM technique, microwave frequency radiation is applied to the tip-sample junction. By exploiting the nonlinear behavior of the junction using high frequencies, ACSTM can be used to acquire capacitance information. Specifically, the tunneling current response at microwave frequencies when imaging semiconductors reflects the $\partial C / \partial V$ data [22]. In this way, ACSTM can provide high resolution spatial capacitance information on semiconducting polymer films. The magnitude of the ACSTM signal ultimately reflects the carrier concentration in the substrate. For example, this microwave signal has been used previously in both STM and AFM mode to measure the dopant concentration in silicon [23-25].

For this ACSTM experiment a loop antenna geometry was used to apply microwave radiation to the junction similar to studies previously reported [27]. We used the difference frequency ACSTM technique [23-26] to simplify detection of the highfrequency modulation of the tunneling signal by allowing the use of conventional lock-in amplifier equipment. Care was taken to ensure that the ACSTM data on both samples were acquired under nearly identical scanning conditions ${ }^{1}$. The ACSTM signal was acquired at each point during scanning, thus producing $\partial C / \partial V$ spectral images (magnitude and phase) and the topography image simultaneously. All scans were taken in ultrahigh vacuum at base pressures on the order of $10^{-10}$ Torr using a commercial RHK STM and mechanically cut Pt:Rh (80:20) tips.

\footnotetext{
${ }^{1}$ Frequencies used were 300 and $300.004 \mathrm{MHz}$, approximately $3.7 \mathrm{dbm}$ for the Pt sample and $3.0 \mathrm{dbm}$ for the Au sample. The difference is due to the change in antenna position when switching between samples, and was adjusted by monitoring the input at the lock-in amplifier to ensure that the initial out-of-tunneling input signal strength at the difference frequency was the same for both samples. The same tip was used for both samples.
} 
The STM was modified to include the antenna so that the loop antenna encircled the STM tip during scanning, similar to a method reported previously [27] and could be moved aside for tip and sample exchange. P3HT films were spin-coated onto Auand $\mathrm{Pt}$-coated $\mathrm{SiO}_{2} / \mathrm{Si}$ substrates following the same procedure as in the fabrication of the OFET devices.

In Fig. 6, the topography and ACSTM images are both shown at various bias conditions for P3HT deposited on $\mathrm{Au}$ and $\mathrm{Pt}$, respectively ${ }^{1}$. The ACSTM images are shown at the same relative color scale in units of $\mathrm{mV}$, directly proportional to local $\partial C / \partial V$ and therefore carrier concentration (as seen in doped Si devices [23]). Bear in mind that the carrier concentration seen in semiconductors in ACSTM depends to some degree on the bias conditions and gap geometry. The gap resistance was kept constant at $\sim 100 \mathrm{G} \Omega$ to eliminate the influence of tip height on the resulting data. A direct comparison of the ACSTM data on $\mathrm{Au}$ and Pt shows a marked contrast in carrier concentration. Averaged over the scan area, the carrier concentration is $25 \%$ larger for $\mathrm{P} 3 \mathrm{HT}$ on $\mathrm{Pt}$ than on $\mathrm{Au}$, but there is also a higher spatial variance on the Pt sample despite the comparable surface structure. This is consistent with the interpretation that there are more mobile holes in the P3HT layer on Pt. Measurements taken at multiple locations on each film confirm these results. In addition, the percentage difference between average ACSTM signal on Pt vs $\mathrm{Au}$ increases to $69 \%$ as the tip bias relative to the sample is increased to $2 \mathrm{~V}$. This shows that the carriers present on $\mathrm{Au}$ are easier to deplete relative to

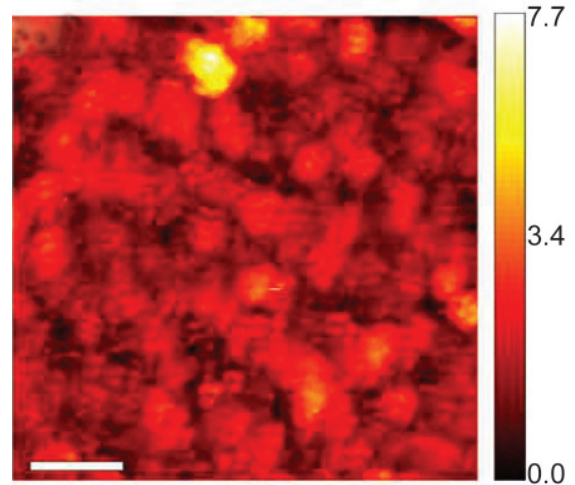

(a)

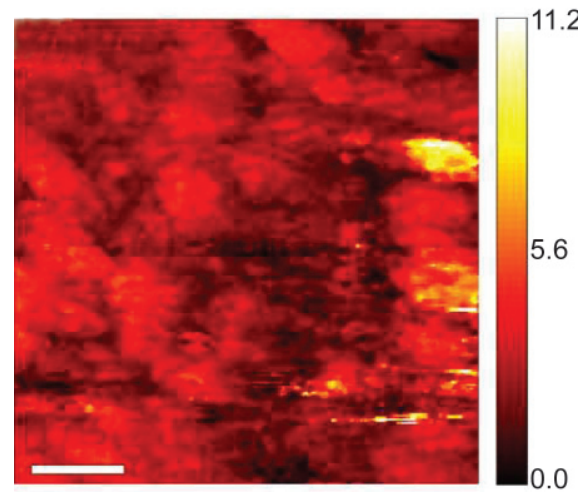

(c)

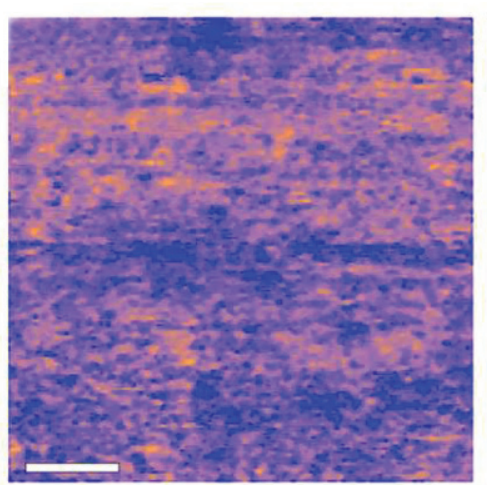

(b)

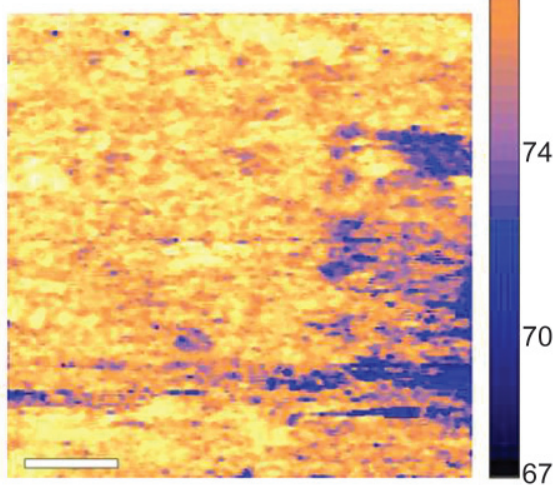

(d)

Figure 6 Topography (a), (c) and ACSTM (b), (d) magnitude images of P3HT films deposited on $\mathrm{Au}(\mathrm{a})$, (b) and Pt (c), (d). The simultaneously acquired ACSTM images are shown at the same relative color scale. All images were acquired at $-1.0 \mathrm{~V}$ sample bias, $10 \mathrm{pA}$ tunneling current, and are $473 \mathrm{~nm} \times 473 \mathrm{~nm}$. The scalebars on all images are $94.6 \mathrm{~nm}$. The color bars are in units of $\mathrm{nm}$ for the topography images and $\mathrm{mV}$ for the ACSTM images. The greater magnitude in (d) indicates an excess of mobile holes for the P3HT/Pt case relative to the P3HT/Au case in (b)

\footnotetext{
${ }^{1}$ Topography images were processed using plane-/offset-subtraction and median filtering in MATLAB, while ACSTM images were only median filtered.
} 
those on Pt. It is difficult to compare quantitatively the ACSTM data in these vertical layered structures with the situation in the transistor geometry. However, the ACSTM data presented here clearly indicate that Pt increases the carrier concentration in the P3HT film by more than the Au layer.

Interestingly, both films show inhomogeneities in the magnitude and phase of their AC response that do not correlate in any immediately obvious way with sample topography, although Figs. 6(a) and 6(c) show that the surface features of the two films are similar. In particular, the variation takes place on length scales that are not correlated with intrinsic structural properties of the underlying polycrystalline metal films, such as grain size.

The transport and ACSTM indications of charge transfer are consistent with simple calculations using a model for charge transfer developed by Paasch and Scheinert [28]. Their model was developed to explain charge transfer and band bending in thin layers of disordered organic semiconductor on top of metal films. This is precisely the situation for the ACSTM samples, and at zero source-drain bias should be reasonably applicable to the organic channel in the immediate vicinity of the source or drain electrodes. Assuming an exponential density of states for the organic semiconductor characterized by an energy scale $k_{\mathrm{B}} T_{0}=0.1 \mathrm{eV}$, the local potential in the organic semiconductor of layer thickness $d$ as a function of distance $x$ away from the metal interface is given by

$$
U(x)=U_{d} \pm k_{\mathrm{B}} T_{0} \ln \left[1+\tan ^{2}\left(\frac{d-x}{2 L_{d}} \exp \frac{\left|U_{d}\right|}{2 k_{\mathrm{B}} T_{0}}\right)\right]
$$

where $U_{d}$ is the potential at $x=d, L_{d} \equiv \sqrt{\varepsilon \varepsilon_{0} k_{\mathrm{B}} T / 2 e^{2} n}$ is the intrinsic screening length, and $n=\sqrt{N_{\mathrm{c}} N_{\mathrm{v}}}$. $\exp \left(-E_{\mathrm{g}} / 2 k_{\mathrm{B}} T_{0}\right)$ is the effective intrinsic carrier density [28]. Here $\varepsilon$ is the relative dielectric constant of the organic semiconductor, $E_{g}$ is the band gap of the organic semiconductor, $N_{\mathrm{c}}$ and $N_{\mathrm{v}}$ are the effective densities of states in the conduction and valence bands. The \pm sign is positive (negative) for hole (electron) accumulation. The Poisson equation can be used to find the relationship between $d$ and $U_{d}$ :

$d=2 L_{d} \exp \left(-\frac{\left|U_{d}\right|}{2 k_{\mathrm{B}} T_{0}}\right) \arctan \left[\exp \left(\frac{\left|U_{\mathrm{s}}-U_{d}\right|}{k_{\mathrm{B}} T_{0}}\right)-1\right]^{0.5}$
Here $U_{\mathrm{s}} \equiv E_{\mathrm{g}} / 2-\Phi_{\mathrm{h}}$, where $\Phi_{\mathrm{h}}$ is the hole injection barrier. Rough values used for our estimations are $N_{\mathrm{c}}$ $N_{\mathrm{v}}=10^{21} \mathrm{~cm}^{-3}, E_{\mathrm{g}}=2 \mathrm{eV}, \varepsilon=3.24$, and $\mathrm{T}=300 \mathrm{~K}$.

Working with Eqs. (1), (2), and Poisson's equation, we can determine the charge density in the organic semiconductor layer. Varying the metals involved affects the local potential $U(x)$ and carrier density by altering $\Phi_{\mathrm{h}}$. Recalling previous photoemission results for P3HT on platinum and gold [16], for platinum with $\Phi_{\mathrm{h}}=0.6 \mathrm{eV}$, the charge carrier density very near the interface is estimated to be $\sim 9.6 \times 10^{17} \mathrm{~cm}^{-3}$; for gold with $\Phi_{\mathrm{h}}=1.2 \mathrm{eV}$, we find $\sim-10^{18} \mathrm{~cm}^{-3}$, with the negative sign indicating a depletion of hole density. These precise numbers should be viewed cautiously, since different surface preparations can alter the effective work functions of metals and the density of states values used are rough. The trend, however, from $\mathrm{Au}$ to $\mathrm{Pt}$ should be robust and these results are unlikely to change qualitatively upon inclusion of corrections for a Gaussian density of states for the polymer. For both the $\mathrm{Pt}$, the $\Phi_{\mathrm{h}}=0.6 \mathrm{eV}$ is consistent with prior photoemission experiments [16] as well as recent Fermi level pinning results for P3HT and estimates of polaron formation energy relevant to $\mathrm{Pt} /$ P3HT charge transfer [6]. The estimate of $\Phi_{\mathrm{h}}$ for $\mathrm{Au}$ also comes from the same photoemission experiments [16]. The resulting calculation supports the claim that higher work function metals, with Fermi levels pinned relatively close to the valence band, locally dope the organic material very near the interface.

We have presented a series of experiments examining the contact resistance and ZGB current in very short channel bottom-contact OFETs incorporating P3HT. The striking differences observed between devices with $\mathrm{Pt}$ and $\mathrm{Au}$ electrodes are consistent with expectations of metal/P3HT charge transfer inferred from photoemission experiments. In particular, it appears that a significant density of mobile holes are transferred from $\mathrm{Pt}$ into neighboring P3HT on the nanoscale. ACSTM measurements further support this conclusion, showing a significantly higher free carrier response in P3HT films on Pt compared to identically prepared films on Au. Further experiments, particularly those probing spatial scales comparable to small numbers of polymer chains, should be able to shed further 
light on the complex problem of charge transfer and Fermi level pinning, complementing spatially averaging techniques such as photoemission. Techniques like ACSTM point the way toward detailed quantitative assessment of local charge density, information that will be extremely useful in refining theoretical models of these important but complicated problems.

\section{Acknowledgments}

The authors gratefully acknowledge Jun Zhang for experimental assistance, Paul Weiss for useful discussions, Prof. J. W. Ciszek and Prof. J. M. Tour for synthesis of the F-OPE molecule, and the support from NSF grant ECCS-0601303. R. Giridharagopal acknowledges the support of an NSF graduate fellowship. D. Natelson also aknowledges the David and Lucille Packard Foundation, the Alfred P. Sloan Foundation, the Robert A. Welch Foundation, and the Research Corporation. K. F. Kelly also acknowledges the Rochester MURI on Nanoscale Subsurface Spectroscopy and Tomography (F49620-031-0379), administered by the Air Force Office of Scientific Research.

\section{References}

[1] Koch, N. Energy levels at interfaces between metals and conjugated organic molecules. J. Phys: Condens. Matter 2008, 20, 184008.

[2] Campbell, I. H.; Rubin, S.; Zawodzinski, T. A.; Kress, J. D.; Martin, R. L.; Smith, D. L.; Barashkov, N. N.; Ferraris, J. P. Controlling Schottky energy barriers in organic electronic devices using self-assembled monolayers. Phys. Rev. $B$ 1996, 54, R14321-R14324.

[3] Nüesch, F.; Rotzinger, F.; Si-Ahmed, L.; Zuppiroli, L. Chemical potential shifts at organic device electrodes produced by grafted monolayers. Chem. Phys. Lett. 1998, 288, 861-867.

[4] Hamadani, B. H.; Corley, D. A.; Ciszek, J. W.; Tour, J. M.; Natelson, D. Controlling charge injection in organic fieldeffect transistors using self-assembled monolayers. Nano Lett. 2006, 6, 1303-1306.

[5] Hill, I. G.; Rajagopal, A.; Kahn, A.; Hu, Y. Molecular level alignment at organic semiconductor-metal interfaces.
Appl. Phys. Lett. 1998, 73, 662-664.

[6] Tengstedt, C.; Osikowicz, W.; Salaneck, W. R.; Parker, I. D.; Hsu C. -H.; Fahlman, M. Fermi-level pinning at conjugated polymer interfaces. Appl. Phys. Lett. 2006, 88, 053502.

[7] Koch N.; Vollmer, A. Electrode-molecular semiconductor contacts: Work-function-dependent injection barriers versus Fermi-level pinning. Appl. Phys. Lett. 2006, 89, 162107.

[8] Vàzquez, H.; Dappe, Y. J.; Ortega, J.; Flores, F. Energy level alignment at metal/organic semiconductor interfaces: "Pillow" effect, induced density of interface states, and charge neutrality level. J. Chem. Phys. 2007, 126, 144703.

[9] Vàzquez, H.; Flores, F.; Oszwaldowski, R.; Ortega, J.; Perez, R.; Kahn, A. Barrier formation at metal-organic interfaces: Dipole formation and the charge neutrality level. Appl. Surf. Sci. 2004, 234, 1-4.

[10]Crispin, A.; Crispin, X.; Fahlman, M.; Berggren, M.; Salaneck, W. R. Transition between energy level alignment regimes at a low band gap polymer-electrode interfaces. Appl. Phys. Lett. 2006, 89, 213503.

[11] Hwang, J.; Kim, E. -G.; Liu, J.; Br'edas, J. -L.; Duggal, A.; Kahn, A. Photoelectron spectroscopic study of the electronic band structure of polyfluorene and fluorenearylamine copolymers at interfaces. J. Phys. Chem. C 2007, 111, 1378-1384.

[12] Bürgi, L.; Richards, T. J.; Friend, R. H.; Sirringhaus, H. Close look at charge carrier injection in polymer fieldeffect transistors. J. Appl. Phys. 2003, 94, 6129-6137.

[13] Li, T.; Ruden, P. P.; Campbell, I. H.; Smith, D. L. Investigation of bottom-contact organic field effect transistors by two-dimensional device modeling. J. Appl. Phys. 2003, 93, 4017-4022.

[14] Hamadani, B. H.; Natelson, D. Nonlinear charge injection in organic field-effect transistors. J. Appl. Phys. 2005, 97, 064508.

[15] Ng, T. N.; Silveira,W. R.; Marohn, J. A. Dependence of charge injection on temperature, electric field, and energetic disorder in an organic semiconductor. Phys. Rev. Lett. 2007, 98, 066101.

[16] Hamadani, B. H.; Ding, H.; Gao, Y.; Natelson, D. Dopingdependent charge injection and band alignment in organic field-effect transistors. Phys. Rev. B 2005, 72, 235302

[17] Cai, L. ; Yao, Y.; Yang, J.; Price, D. W., Jr.; Tour, J. M. 
Chemical and potential assisted assembly of thioacetylterminated oligo(phenylene ethynylene)s on gold surfaces. Chem. Mater. 2002, 14, 2905-2909.

[18] Hamadani, B. H.; Natelson, D. Temperature-dependent contact resistances in high quality polymer field-effect transistors. Appl. Phys. Lett. 2004, 84, 443-445.

[19] Gundlach, D. J.; Royer, J. E.; Park, S. K.; Subramanian, S.; Jurchescu, O. D.; Hamadani, B. H.; Moad, A. J.; Kline, R. J.; League, L. C.; Kirillov, O.; Richter, C. A.; Kushmerick, J. G.; Richter, L. J.; Parkin, S. R.; Jackson, T. N.; Anthony, J. E. Contact-induced crystallinity for high-performance soluble acene-based transistors and circuits. Nat. Mater. 2008, 7, 216-221.

[20] Merlo, J. A.; Frisbie, C. D. Field effect conductance of conducting polymer nanofibers. J. Polym. Sci. B: Polym. Phys. 2003, 41, 2674-2680.

[21] Rep, D. B. A.; Morpurgo, A. F.; Klapwijk, T. Dopingdependent charge injection into regioregular poly(3hexylthiophene). Org. Electron. 2003, 4, 201-207.

[22] Bourgoin, J. P.; Johnson, M. B.; Michel, B. Semiconductor characterization with the scanning surface harmonic microscope. Appl. Phys. Lett. 1994, 65, 2045-2047.

[23] Donhauser, Z. J.; McCarty, G. S.; Bumm, L. A.; Weiss P. S. High resolution dopant profiling using a tunable AC scanning tunneling microscope. In Characterization and Metrology for ULSI Technology: 2000 International
Conference; Seiler, D. G. et al., Eds.; American Institute of Physics: New York, 2001, pp. 641-646.

[24] Kelly, K. F.; Donhauser, Z. J.; Mantooth, B. A.;Weiss, P. S. Expanding the capabilities of the scanning tunneling microscope. In NATO ASI Series II: Mathematics, Physics, and Chemistry; Vilarinho, P.; Rosenwaks, Y.; Kingon, A., Eds.; Springer: New York, 2005, pp. 153-171.

[25] Schmidt, J.; Rapoport, D. H.; Fröhlich, H. -J. Microwavefrequency alternating current scanning tunneling microscopy by difference frequency detection: Atomic resolution imaging on graphite. Rev. Sci. Instr. 1999, 70, 3377-3380.

[26] Bumm, L. A.; Arnold, J. J.; Cygan, M. T.; Dunbar, T. D.; Burgin, T. P. ; Jones, L. II,; Allara, D. L.; Tour, J. M.; Weiss, P. S. Are single molecular wires conducting? Science 1996, 271, 1705-1707.

[27] Lee, J.; Tu, X.; Ho, W. Spectroscopy and microscopy of spin-sensitive rectification current inducted by microwave radiation. Nano Lett. 2005, 5, 2613-2617.

[28] Paasch, G.; Scheinert, S. Space charge layers in organic field-effect transistors with Gaussian or exponential semiconductor density of states. J. Appl. Phys. 2007, 101, 024514.

[29] Fursina, A.; Lee, S.; Sofin, R. G. S.; Shvets, I. V.; Natelson, D. Nanogaps with very large aspect ratios for electrical measurements. Appl. Phys. Lett. 2008, 92, 113102. 